\title{
Review
}

\section{Controlling the Performance of Filled Rubbers}

\author{
Seiichi KAWAHARA ${ }^{* \dagger}$, Yoshimasa YAMAMOTO**, and Yoshinobu ISONO* \\ "Department of Materials Science and Technology, Faculty of Engineering, \\ Nagaoka University of Technology, \\ 1603-1 Kamitomioka-cho, Nagaoka, Niigata 940-2188, Japan \\ ${ }^{* *}$ Department of Materials Science and Engineering, \\ Tokyo National College of Technology, \\ 1220-2, Kunugida-machi, Hachioji, Tokyo 193-0997, Japan \\ (Received : October 30, 2013)
}

\begin{abstract}
Performance of filled rubber, which depended on the application such as tires, engine mounts, belts, seismic isolation rubber and rubber rollers, was described in conjunction with properties, i.e. strength, elasticity, viscosity, hardness, durability, weather resistance, conductivity, etc. Factors to control the properties were discussed in detail, which was associated with the amount of filler, the primary structure of filler, the secondary structure of its aggregate, and its distribution. In particular, the properties were related to rubber-rubber, rubber-filler and filler-filler interactions, which were inherently held in the filled rubber. The state of art technology and evolutionary prospect were described to control performance of tires, engine mounts, belts, seismic isolation rubber, rubber rollers and so forth.
\end{abstract}

Key Words: Rubber / Carbon black

\section{INTRODUCTION}

The performance requirements for filled rubber greatly depend on the application (i.e., tires, engine mounts, belts, seismic isolation rubber, rubber rollers, etc.). Table I shows some examples. Thus, universal filled rubber, which possesses all the properties required for high performance products, is difficult to prepare, especially since some properties are antonymous. Additionally, the performance of filled rubber depends significantly on both the filler and filler aggregate

Table I. Properties as performance indices for rubber products.

\begin{tabular}{|l|l|}
\hline Products & Properties as performance indices \\
\hline Tires & $\begin{array}{l}\text { high tensile strength, high tear strength, high rebound } \\
\text { resilience, high wet skid resistance, low heat build- } \\
\text { up, high handling stability, comfort driving }\end{array}$ \\
\hline Engine mounts & $\begin{array}{l}\text { high tensile strength, high tear strength, low ratio of } \\
\text { dynamic to static modulus, high damping properties, } \\
\text { high heat resistance, good durability }\end{array}$ \\
\hline Belts & $\begin{array}{l}\text { high tensile strength, high tear strength, high bending } \\
\text { resistance, high wear resistance }\end{array}$ \\
\hline $\begin{array}{l}\text { Seismic } \\
\text { isolation rubber }\end{array}$ & $\begin{array}{l}\text { high tensile strength, high tear strength, high shear spring } \\
\text { constant, high damping properties, good durability }\end{array}$ \\
\hline Rubber rollers & $\begin{array}{l}\text { high tensile strength, high tear strength, high wear } \\
\text { resistance, good durability, high electrical conductivity }\end{array}$ \\
\hline
\end{tabular}

$\dagger$ E-mail: kawahara@mst.nagaokaut.ac.jp structure. Consequently, high performance filled rubber is designed specifically for each product.

Filled rubber is a composite material consisting of rubber and filler. In addition to selecting a suitable combination of rubber and filler, it is important to control the primary structure of the filler, the secondary structure of its aggregate, and its distribution. In particular, the appropriate processing conditions are indispensable to enhance the performance of filled rubber because the secondary structure of the aggregates and the distribution of the filler and/or its aggregates depend upon the structure of the filler ${ }^{1)}$ and the processing conditions. ${ }^{2)}$ Thus, precisely controlling the non-equilibrium structures of filled rubber governs performance. In this review article, methods to prepare high-performance filled rubber are described in terms of the relationship between the structure and properties of the filled rubber.

\section{FILLER EFFECTS}

The filler effects on the properties of filled rubber can be divided into three categories ${ }^{3,4)}$ : volume, interface, and cavitation effects. The volume effect is proportional to the volume fraction of the filler, while the interface effect is associated with the interactions between the rubber and the filler, and the cavitation effect is related to the presence of an interfacial cavity or void between the rubber and the filler. 
The volume and the interface effects may play important roles in improving and reinforcing the properties of filled rubber, whereas the cavitation effect reduces the properties due to propagation of the generated cracks (Figure 1). Furthermore, the morphology also influences the properties. Because each effect has a unique origin, it is necessary to understand all of them.

\subsection{Volume Effect}

The volume effect is due to the nature of a heterogeneous system that consists of rubber as a medium and filler as a dispersoid. Because rubber is viscoelastic material, when the filler is composed of rigid, spherical particles, the viscosity $(\eta)$ and modulus $(E)$ are expressed by the Guth-Gold ${ }^{5)}$ and Smallwood $^{(6)}$ equations, respectively.

$$
\begin{aligned}
& \eta=\eta_{0}\left(1+2.5 c+14.1 c^{2}\right), \\
& E=E_{0}\left(1+2.5 c+14.1 c^{2}\right),
\end{aligned}
$$

where $\eta_{0}$ and $E_{0}$ are the viscosity and the modulus of neat rubber, and $c$ is the volume fraction of the filler. If the particles are not spherical, the modulus is expressed by the following equation using a shape factor. ${ }^{7}$

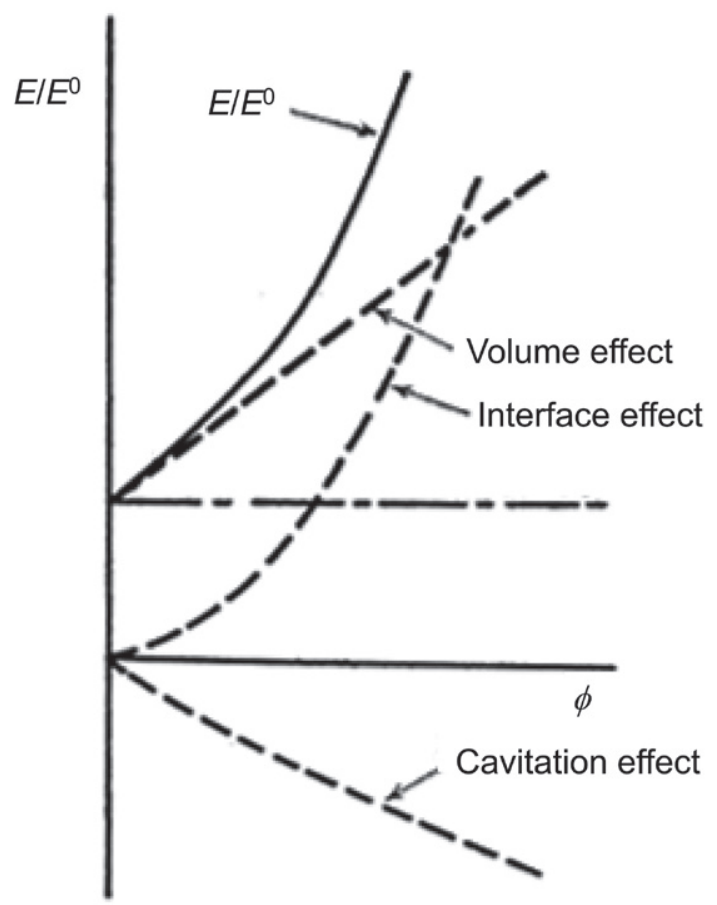

Fig. 1. Volume, interface, and cavitation effects on Young's modulus of an filled rubber, which is normalized with Young's modulus of rubber, itself. ${ }^{4}$, Copyright $(1066$ The Society of Rubber Science and Technology, Japan.

$$
E=E_{0}\left(1+0.67 f c+1.62 f^{2} c^{2}\right),
$$

where $\mathrm{f}$ is a shape factor defined as a length/width ratio of the particle. Equation (2) is held at $\mathrm{f}>>1$. For filler having a large aspect ratio L/D, the following Halpin-Tsai equation ${ }^{7)}$ (4) is adopted instead of equation (2), in which $L$ is length of the filler and D is its diameter.

$$
\begin{aligned}
& E_{L}=E_{m} \frac{1+\xi \eta \phi}{1-\eta \phi} \text { and } E_{T}=E_{m} \frac{1+\eta \phi}{1-\eta \phi} \\
& \eta=\frac{\left(E_{f} / E_{m}\right)-1}{\left(E_{f} / E_{m}\right)+\xi} \text { with } \xi=2\left(\frac{L}{D}\right)
\end{aligned}
$$

Here, $E_{f}$ and $E_{m}$ represent the moduli of the filler and rubber, respectively. The $E_{L}$ and $E_{T}$ represent longitudinal modulus and transverse modulus of the filled rubber, whose directions are defined to be parallel and perpendicular to the fiber axial direction of the filler, respectively. The value of the modulus agrees well with equation (2) for rubber filled with glass beads and medium thermal blacks, but exceeds the value estimated by equation (2) with carbon black holding a large surface area. Figure 2 shows the relationship between the volume fraction of carbon black $(\phi)$ and the Young's modulus $(E)$ of styrenebutadiene rubber (SBR) filled with carbon black ${ }^{9)}$, in which closed circles with error bar represent experimentally obtained results and broken line represents theoretical value estimated by equation (2). The value of the Young's modulus of SBR increases as the volume fraction of carbon black increases, which is consistent with the value estimated by equation (2), implying that the volume effect of carbon black is attributed to the spherical particles present in the rubber medium.

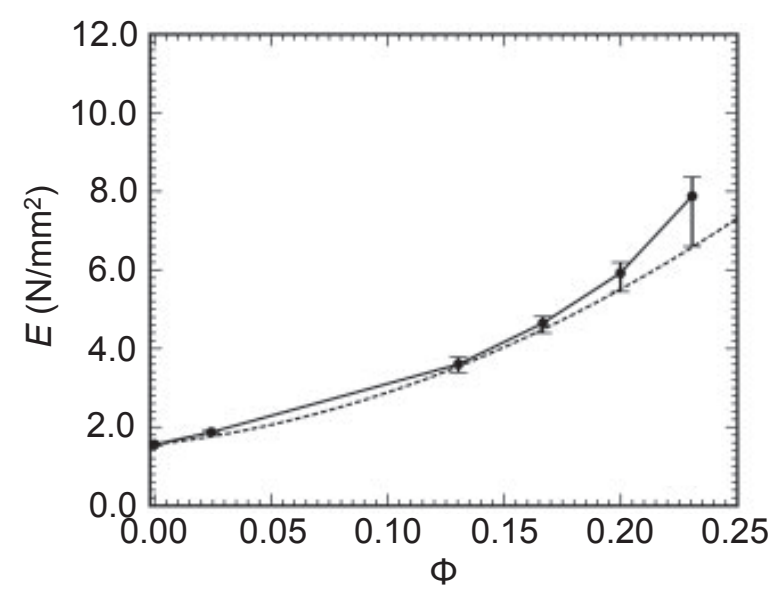

Fig. 2. Relationship between the volume fraction of carbon black and the Young's modulus of styrene-butadiene rubber (SBR) filled with carbon black. ${ }^{9}$, Copyright (C) 2013 Wiley Periodicals, Inc. 


\subsection{Interface Effect}

Because the interface effect depends on the physical and chemical interactions between the rubber and the filler ${ }^{10}$, it is related to the physical and chemical adsorptions of the rubber onto the surface of the filler ${ }^{11,12}$, surface roughness of the filler $^{13)}$, surface area ${ }^{14)}$, etc. In particular, the properties of filled rubber are improved as the amount of the rubber bound onto the surface of the filler increases; that is, the higher the amount of bound rubber, the better the properties. Figure 3 and 4 plot the Mooney viscosity versus the volume fraction of bound rubber for styrene-butadiene rubber (SBR) and butadiene rubber, which are filled with carbon black. ${ }^{15)}$ The value of the

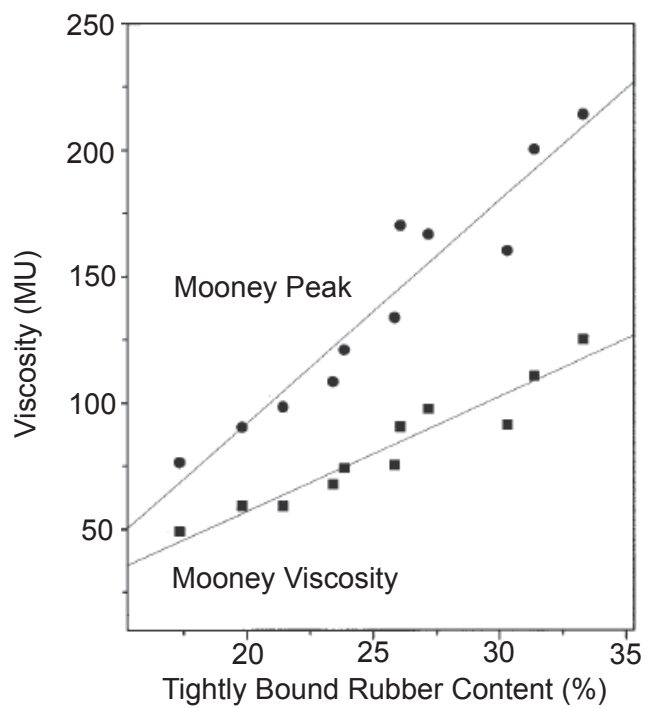

Fig. 3. Mooney viscosity versus the volume fraction of bound rubber for the filled SBR, in which the carbon blacks used are various amount of N326, N330 and N351. ${ }^{15)}$, Copyright (C) 2004 Wiley Periodicals, Inc.

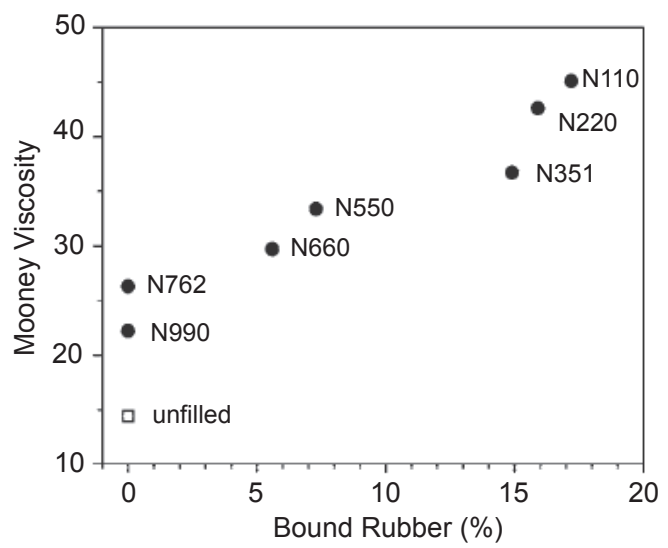

Fig. 4. Mooney viscosity versus the volume fraction of bound rubber for butadiene rubber, in which the carbon blacks $(0.18$ in the volume fraction) used are listed in Table II. ${ }^{15)}$, Copyright (C) 2004 Wiley Periodicals, Inc.
Mooney viscosity increases as the volume fraction of bound rubber increases, indicating that the interface effect may play an important role in reinforcement.

The interface effect is also related to the dissipation of mechanical energy in filled rubber. ${ }^{16)}$ For instance, due to friction between the rubber and the filler at the interface during deformation and reversion, mechanical energy is converted into thermal energy when the rubber is insufficiently attached to the filler. In this case, the values of the loss modulus and loss tangent are higher than those of rubber itself. Figure 5 plots the storage modulus and loss tangent versus the volume fraction of bound rubber ${ }^{17}$ for the filled butadiene rubber, where the carbon blacks used are listed in Table II. For a given amount of filler ( 0.18 in the volume fraction), the storage modulus decreases, but the loss modulus and loss tangent increase, as the volume fraction of bound rubber decreases.

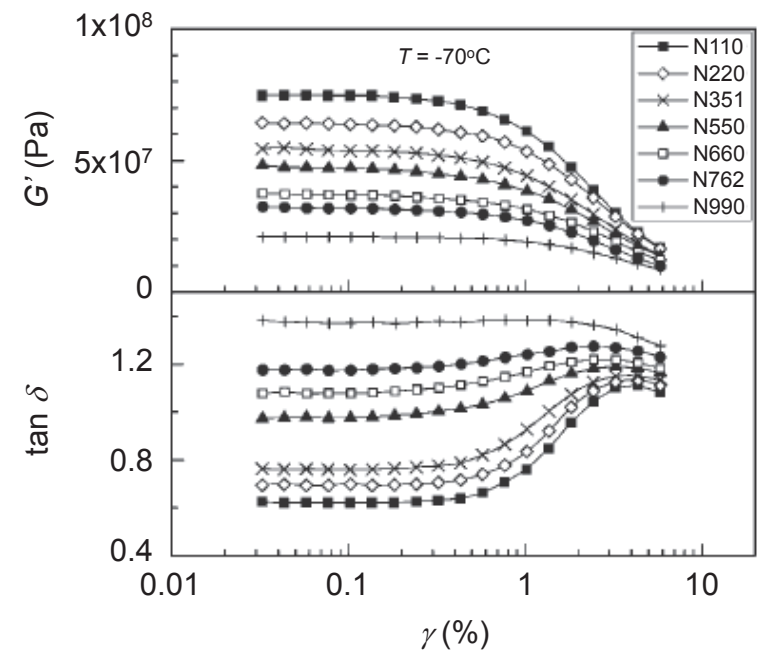

Fig. 5. Storage modulus and loss tangent versus the volume fraction of bound rubber for the filled butadiene rubber, in which the carbon blacks used are listed in Table II. ${ }^{17}$ ), Copyright 2008 American Chemical Society.

Table II. Characteristics of carbon blacks. ${ }^{17)}$

\begin{tabular}{cccc}
\hline Carbon black type & $d(\mathrm{~nm})$ & $D(\mathrm{~nm})$ & $S\left(\mathrm{~m}^{2} / \mathrm{g}\right)$ \\
\hline N110 & $17 \pm 7$ & $54 \pm 26$ & 143 \\
N220 & $21 \pm 9$ & $65 \pm 30$ & 117 \\
N351 & $31 \pm 14$ & $89 \pm 47$ & 75 \\
N550 & $53 \pm 28$ & $139 \pm 71$ & 41 \\
N660 & $63 \pm 36$ & $145 \pm 74$ & 34 \\
N762 & $110 \pm 53$ & $188 \pm 102$ & 21 \\
N990 & $246 \pm 118$ & $376 \pm 152$ & 9 \\
\hline
\end{tabular}

$d:$ Primary particle diameter

$D:$ Particle aggregate diameter

$S:$ Specific surface area 
Additionally, interactions between filler particles (fillerfiller interactions) may play an important role improving the properties and energy dissipation. Filler particles may agglomerate to form secondary aggregates, which threedimensionally connect to form a filler network ${ }^{18-20)}$ that increases the tensile strength and modulus. However, the filler network ${ }^{21,22)}$ is easily broken into small aggregates after deformation because the filler-filler interaction is too weak to maintain the network structure. The Peyne effect ${ }^{23)}$ and Mullins effect ${ }^{24)}$ are shown in Figures 6 and 7, respectively. The storage modulus and phase $(\delta)$ depend on the strainamplitude (Figure 6), whereas the stress depends on the number of repeating deformations (Figure 7).

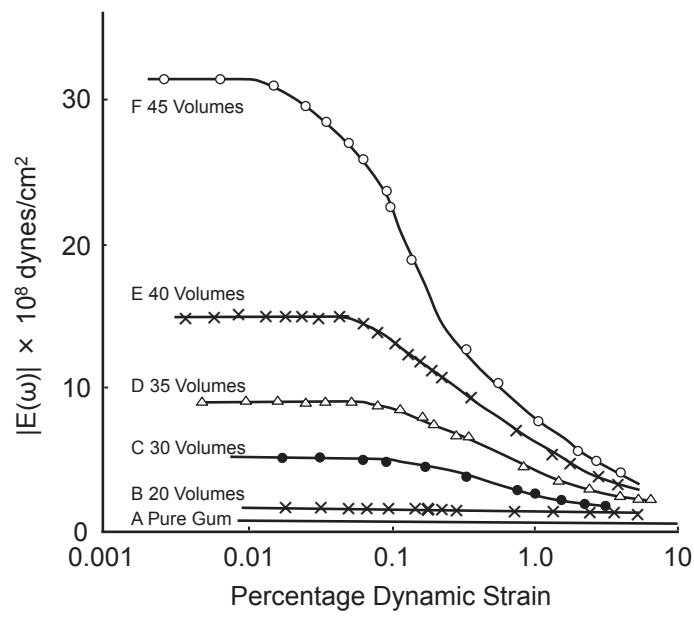

Fig. 6. Plot of complex modulus versus the strain-amplitude (Payne effect) ${ }^{23)}$, Copyright (c) 1963 John Wiley \& Sons, Inc.

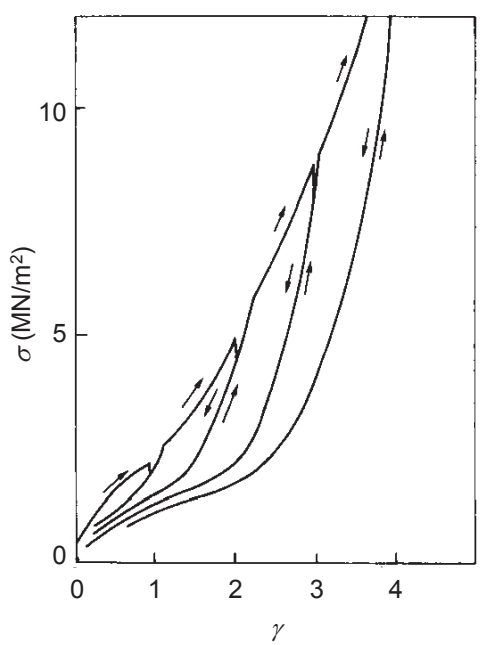

Fig. 7. Stress-strain curves for the filled SBR. The stress depends on the number of repeating deformations (Mullins effect). ${ }^{24)}$, Copyright (C) 1969, Rubber Division, American Chemical Society, Inc.John Wiley \& Sons, Inc.

\subsection{Cavitation Effect}

The cavitation effect, which is a failure in the vicinity of interface between rubber as a medium and filler as a dispersoid ${ }^{25-30)}$, typically originates from cavitation and flaw generation. The cavitation effect may play a negative role in the properties of filled rubber. An interfacial failure between SBR and MT carbon occurs, forming a cavity when the filled rubber is stretched. ${ }^{31)}$ In contrast, a cavity is not formed between SBR and High Abrasion Furnace Black (HAF). SBR is effectively reinforced with HAF, but not with MT carbon. In contrast, the cavitation effect improves the tensile strength (Figure 8). ${ }^{32)}$ Thus, cavities may alter the local stress state and promote the local shear motion of the fillers.

\subsection{Morphology}

Fillers are usually dispersed in a rubber matrix (i.e., an island-matrix structure). They may form the filler network, when the volume fraction of filler increased. Figure 9 shows transmission electron micro tomography (TEMT) images for natural rubber filled with the carbon black. ${ }^{33)}$ The fillers are well dispersed in the rubber, when the fraction is $10 \mathrm{phr}$. In contrast, they gather to form the filler network, when the
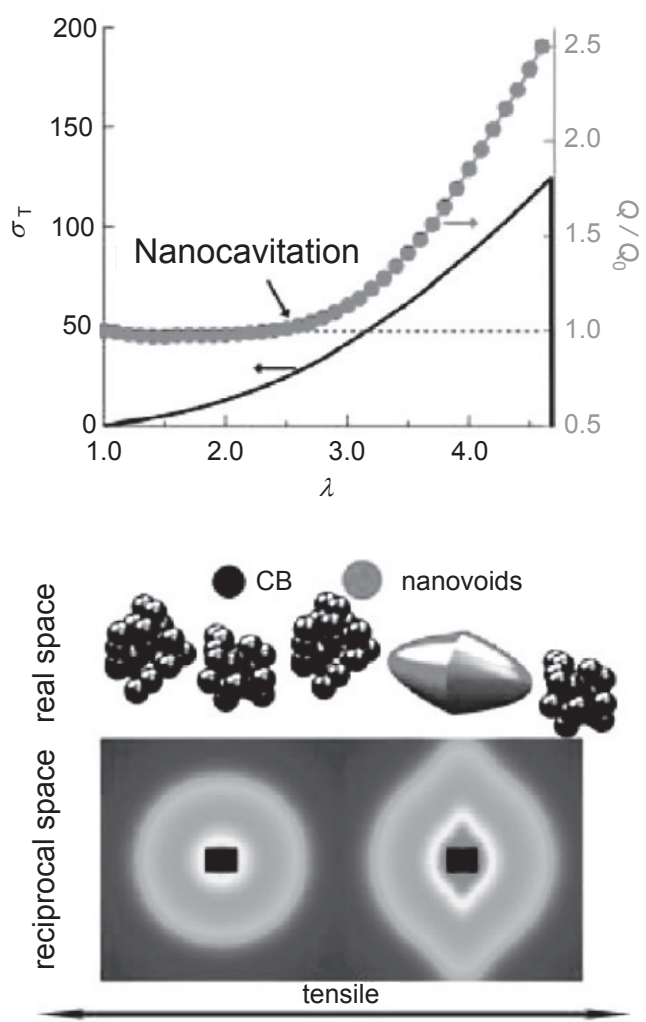

Fig. 8. Scattering and tensile testing data for the carbon black filled SBR. The stress $\left(\sigma_{\mathrm{T}}\right.$ : line $)$ and normalized invariant $\left(Q / Q_{0}\right.$ : filled circle) are presented as a function of elongation $(\lambda) .^{32)}$, Copyright 2012 American Chemical Society. 
fraction is $40 \mathrm{phr}$. The aggregates are linked by the distance between carbon black aggregate within ca. $1 \mathrm{~nm}$ which is the resolution of a TEMT technique and indicate the adjacent carbon black aggregates in different color. The dispersion of the fillers is associated with the mechanical properties of the filled rubber. Thus, the dispersion is considered to be a key factor to control the properties.

Recently, a nanomatrix structure has been proposed as an attractive nanostructure to control the viscoelastic properties $^{34,35)}$, on the basis of the discovery of naturally occurring inhomogeneous structure of natural rubber. ${ }^{36,37)}$ The storage modulus, loss modulus, and loss tangent versus
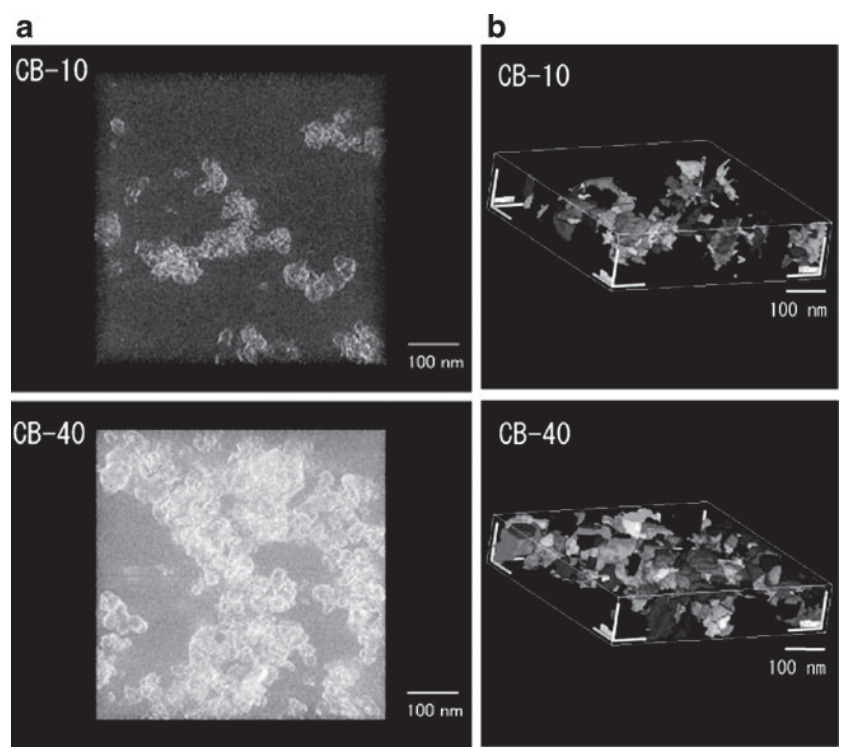

Fig. 9. TEMT images (a) and analysis results (b) of CB-10 and CB-40. In Fig. 9 (b), the aggregates are linked by the distance between $\mathrm{CB}$ aggregate within ca. $1 \mathrm{~nm}$ which is the resolution of a TEMT images and indicate the adjacent $\mathrm{CB}$ aggregates in different color. $^{33)}$, Copyright (C) 2013, Springer Science + Business Media.

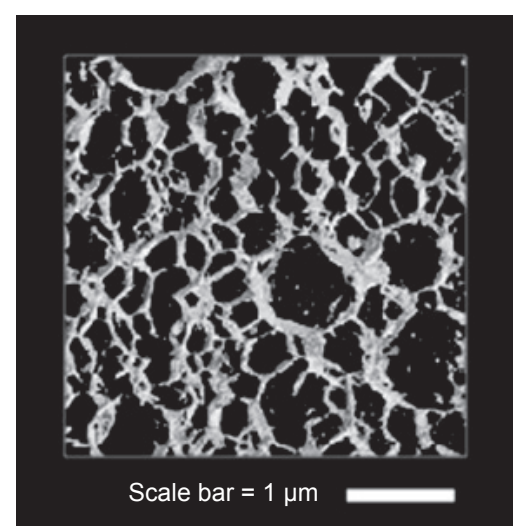

Fig. 10. TEMT image for natural rubber with the nanomatrix structure. The image is taken for natural rubber grafted with polystyrene as a model of the filled rubber with the nanomatrix structure. ${ }^{34)}$, Copyright 2008 American Chemical Society. frequency are individually changed by adjusting the thickness of the matrix, the size of nanoparticles dispersed in the nanomatrix, the distance between the nanoparticles, etc. The nanomatrix structure consists of rubber particles with several$\mu \mathrm{m}$ diameters as the major component and nanoparticles with several tens-nm diameters as the minor component (Figure 10). The nanoparticles densely gather to form a nanomatrix in which the rubber particles are dispersed.

Figure 11 shows the storage modulus in the rubbery plateau region (plateau modulus) versus frequency for natural rubber, rubber with a nanomatrix structure, and rubber with an islandmatrix structure. The plateau-modulus of natural rubber increases about ten times when the nanomatrix structure is formed. In contrast, when natural rubber forms the islandmatrix structure, the plateau-modulus increases slightly (i.e. about 1.3 times as high as that of natural rubber). The large increase in the plateau-modulus is an advantage of the nanomatrix structure.

The loss tangent, which is the ratio of the loss modulus to the storage modulus, is also controlled by the nanomatrix structure. Figure 12 shows the loss tangent of natural rubber and the rubber with a nanomatrix structure in the plateau region. The loss tangent of natural rubber is between 0.1 and 0.15 , depending on the frequency; that is, the higher the frequency, the lower the value of the loss tangent. This may be explained by the viscoelasticity of the rubber. In contrast, when the nanomatrix structure is formed, the loss tangent is independent of the frequency. It is evident that the viscoelastic properties are well controlled by the nanomatrix structure.

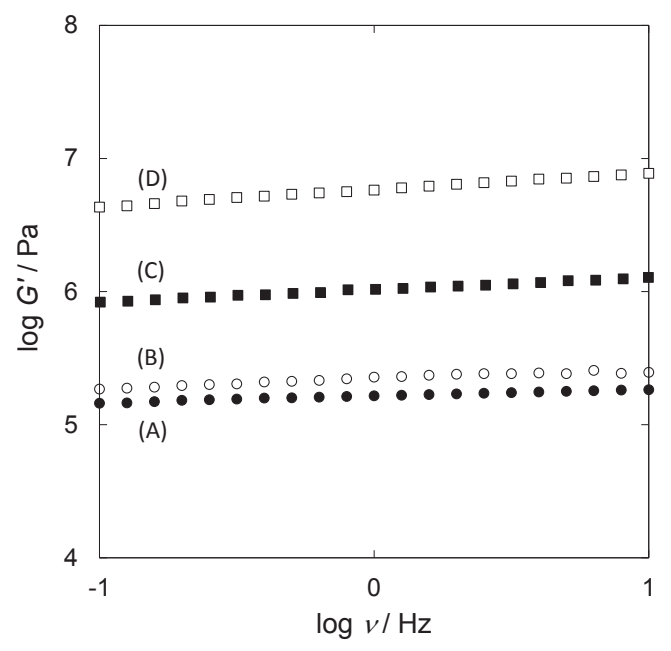

Fig. 11. Storage modulus in the rubbery plateau region (plateau modulus) versus frequency for (A) natural rubber, (B) rubber with an island-matrix structure, $(C)$ rubber with a nanomatrix structure and (D) rubber with a nanomatrix structure annealed at $130{ }^{\circ} \mathrm{C}^{34)}$, Copyright 2008 American Chemical Society. 


\section{NATURAL RUBBER AND SYNTHETIC RUBBER}

The effects of filler greatly differ between natural rubber and synthetic rubber because natural rubber is a straininduced crystallizable polymer, whereas synthetic rubber is a viscoelastic polymer. Figure 13 shows the dependence of the tear energy on the strain rate and temperature for natural rubber and SBR. ${ }^{38)}$ The tear energy of SBR depends significantly on the strain rate and temperature; the higher the temperature and the lower the strain rate, the lower the strain energy. In contrast, the tear energy of natural rubber is quite high compared to that of synthetic rubber due to the effect of strain-induced crystallization, but is almost independent of the strain rate and temperature. ${ }^{39)}$ The effect of strain-induced crystallization on the properties is more remarkable in the tensile strength because the value of the stress at the break of natural rubber is dramatically reduced above the melting

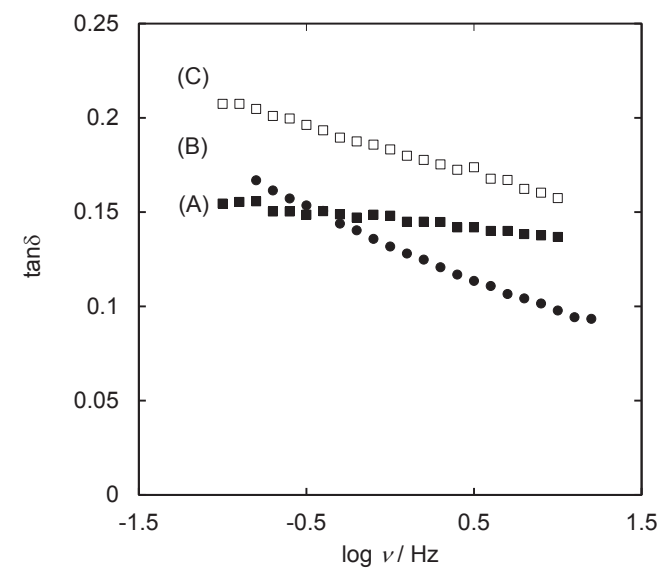

Fig. 12. Loss tangent in the plateau region versus frequency for (A) natural rubber, $(\mathrm{B})$ the rubber with a nanomatrix structure and (C) the rubber with a nanomatrix structure annealed at $130^{\circ} \mathrm{C}^{34)}$, Copyright 2008 American Chemical Society.

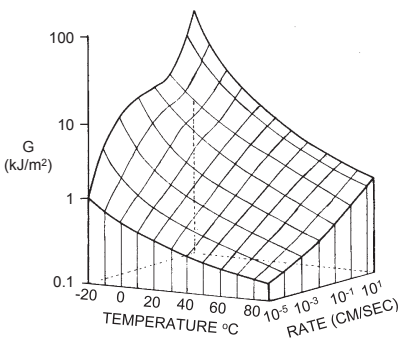

(A)

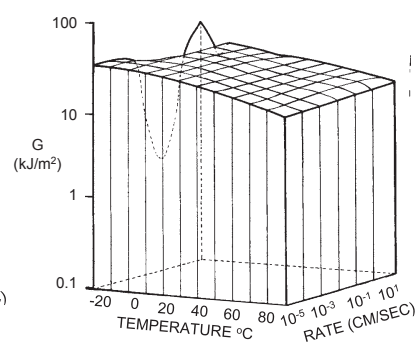

(B)
Fig. 13. Dependence of the tear energy on the strain rate and temperature for (A) SBR and (B) natural rubber. ${ }^{38)}$, Copyright (C) 1955 Interscience Publishers, Inc., New York. temperature of the strain-induced crystal (Figure 14). ${ }^{40)}$ Strain-induced crystallization plays an important role in the mechanical properties of filled natural rubber (Figure 15). The value of the stress at the break of the filled rubber is dramatically reduced above the melting temperature of the strain-induced crystal. ${ }^{41)}$ Although fillers cause volume, interface, and cavitation effects on both natural and synthetic rubbers, fillers also induce a strain-induced crystallization effect in natural rubber.

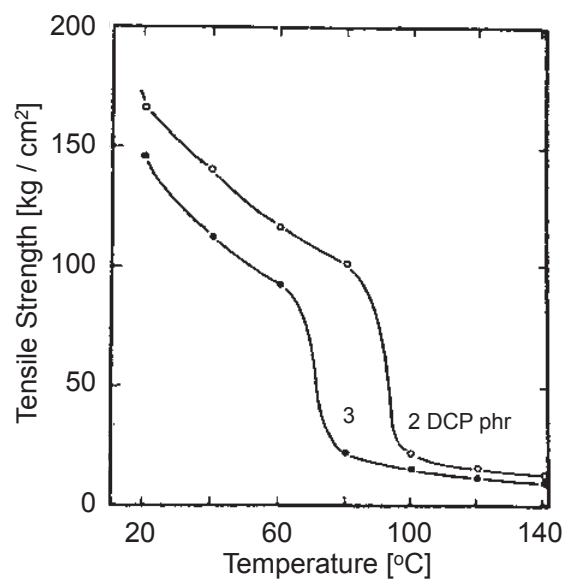

Fig. 14. Plot of tensile strength versus temperature for natural rubber crosslinked with 2 and 3 phr dicumyl peroxide (DCP) ${ }^{40)}$, Copyright (C) 1970, Rubber Division, American Chemical Society, Inc.John Wiley \& Sons, Inc.

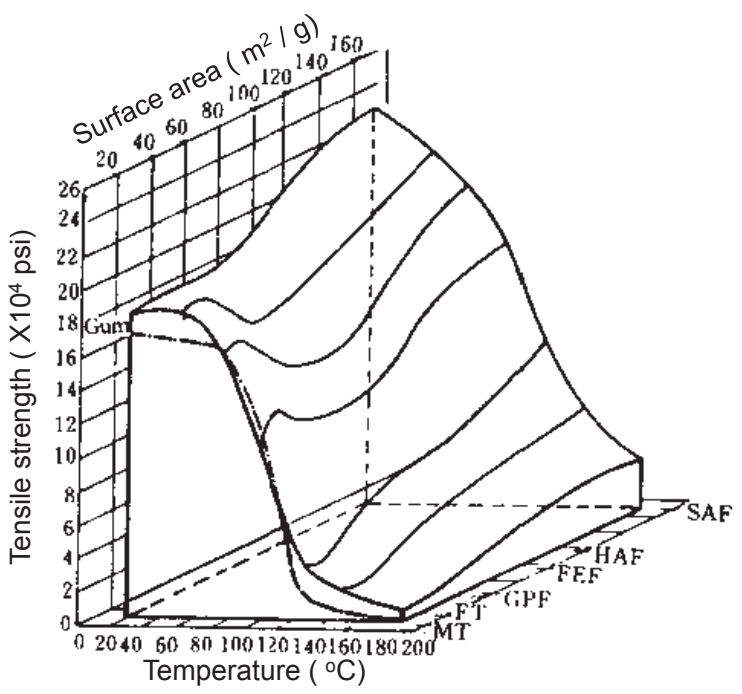

Fig. 15. Temperature dependence of tensile strength of natural rubber filled with various carbon blacks: i.e. Medium Thermal Black (MT), Fine Thermal Black (FT), General Purpose Furnace Black (GPF), Fast Extruding Furnace Black (FEF), High Abrasion Furnace Black (HAF) and Super Abrasion Furnace Black (SAF). ${ }^{41)}$, Copyright (C) 1971, Rubber Division, American Chemical Society, Inc.John Wiley \& Sons, Inc. 


\section{PERFORMANCE OF FILLED RUBBER}

Although the performance varies significantly by application (Table I), it is well associated with the viscoelastic properties and morphology. For instance, to control the performance, the elasticity and the viscosity must be optimally balanced and the filler must be homogeneously dispersed to suppress stress from concentrating at a large deformation. Furthermore, the demand on the energy dissipation is complicated; for instance, energy dissipation must be low for a high rebound resilience and low heat buildup, but it must be high for a high wet skid resistance, high damping properties, and high wear resistance. Consequently, it is crucial to understand the effects of the filler and to develop a suitable filled rubber to meet specific needs.

\subsection{High Strengths}

The tear strength and tensile strength are related to both storage and release of the strain energy of the rubber. A change in force versus time for the tear test, which delineates a locus of a saw tooth ${ }^{42)}$, is a typical example of storage and release of the strain energy. An increase in the force (i.e., tear energy) is related to Young's modulus of the rubber. ${ }^{43)}$ The higher Young's modulus, the higher the tear energy is. In the case of filled rubber, the values of the tear energy and the modulus increase in terms of the volume fraction of the filler according to equations (2), (3), and (4). Thus, the tear strength of filled synthetic rubber is concerned with not only the viscoelastic properties of the rubber ${ }^{44)}$, but also the volume, interface, and cavitation effects of the filler. The same effects influence the tensile strength of filled synthetic rubber. The tear strength and tensile strength likely peak as the filler is homogeneously dispersed into synthetic rubber. ${ }^{45)}$ In contrast, the tear strength and tensile strength of natural rubber are affected by the viscoelastic properties and strain-induced crystallization of the rubber as well as the volume, interface, and cavitation effects of the filler. The strain-induced crystallization of natural rubber is promoted by the stress concentration of the filler. ${ }^{41)}$

\subsection{High Shear Spring Constant}

The shear spring constant is proportional to the shear modulus, which, in turn, is proportional to the longitudinal length and the reciprocal cross-sectional area of the rubber. To increase the shear spring constant, rubber composites contain a hard material to form lamellar or cylinder structures ${ }^{46)}$, which are aligned parallel to the longitudinal direction. In this case, the value of the shear spring constant increases in proportion to the cross-sectional area of the hard material and as a function of the volume fraction of the filler. The increase is more significant when the longitudinally aligned filler domains are well connected.

\subsection{High Rebound Resistance and Low Heat Buildup}

Rebound resilience is high when the dissipation is sufficiently low. ${ }^{47)}$ When the strain energy stored during deformation is released, a high rebound resilience is achieved. In this case, the heat buildup is quite low due to less transfer of mechanical energy to heat energy. A perfect elastic body is an ideal material, which may realize a high rebound resistance and low heat buildup. In contrast, filled rubber is a viscoelastic material, which possesses elasticity and viscosity, making it difficult to eliminate energy dissipation.

Therefore, the elasticity must be increased, while the viscosity must be decreased. For example, the storage modulus increases but the loss modulus decreases in the rubbery plateau region. The storage modulus of filled rubber increases as more filler is added, similar to the loss modulus case. A high storage modulus is required in conjunction with a low loss modulus. Thus, it is necessary to control the anisotropy relaxation of the rubber, friction between rubber particles, friction between rubber and filler particles, friction between filler particles, etc. Here, the friction between filler particles is adjusted by the filler dispersion. A homogeneous dispersion may help prevent an uncertain energy dissipation ${ }^{41)}$ because it increases the distance between filler particles.

\subsection{High Wet Skid Resistance}

The wet skid resistance is directly related to the energy dissipation of a filled rubber. A large energy dissipation at the breaking frequency is required for a high wet skid resistance, which prevents slippage of a tire on a wet road. Increasing the wet skid resistance may reduce the number of car accidents. To control the wet skid resistance, the time-temperature superposition rule of the viscoelastic properties is applied. The glass transition is useful for an index of energy dissipation. The energy loaded during a deformation is spent to change the conformation of the rubber. Using a suitable rubber, which possesses a glass transition temperature in the vicinity of $-30{ }^{\circ} \mathrm{C}^{47)}$ (Figure 16), realizes a high wet skid resistance.

\subsection{High Damping Properties}

The damping properties increase as the energy dissipation increases. To realize high damping properties, the interactions between the filler and rubber and the interactions between the fillers ${ }^{48)}$ must be increased. Thus, the damping properties may be enhanced with insufficient adsorption of the rubber onto the filler, which increases the friction between rubber and filler 
particles and transforms mechanical energy to heat energy. The damping properties are also related to both the relaxation of the anisotropy after deformation and decomposition of the filler aggregates, which increase energy dissipation.

\subsection{Low Ratio of Dynamic to Static Moduli and High Damping Properties}

The ratio of dynamic to static moduli is an index to express the dependence of the modulus of the filled rubber on the frequency. Because this ratio is estimated from the values of the complex moduli at low and high frequencies, to attain a low ratio (ca. 1) of dynamic to static moduli and the high damping properties, the complex modulus must be definite. However, in practice, a low ratio of dynamic to static moduli is not compatible with high damping properties. ${ }^{49)}$ In the rubbery plateau region, the storage modulus increases and the loss modulus decreases as the frequency increases. One solution for this contradiction is to control the dispersion of the filler in the rubber (e.g., individual controls on the storage modulus and the loss modulus). The nanomatrix structure may be useful to achieve a low ratio of dynamic to static moduli and high damping properties. ${ }^{34)}$

\subsection{High Wear Resistance}

The wear resistance relies on the flexibility of filled rubber to resist tearing due to force and heat. A large energy dissipation and large volume to prevent a local stress from concentrating are required for a high wear resistance ${ }^{50)}$

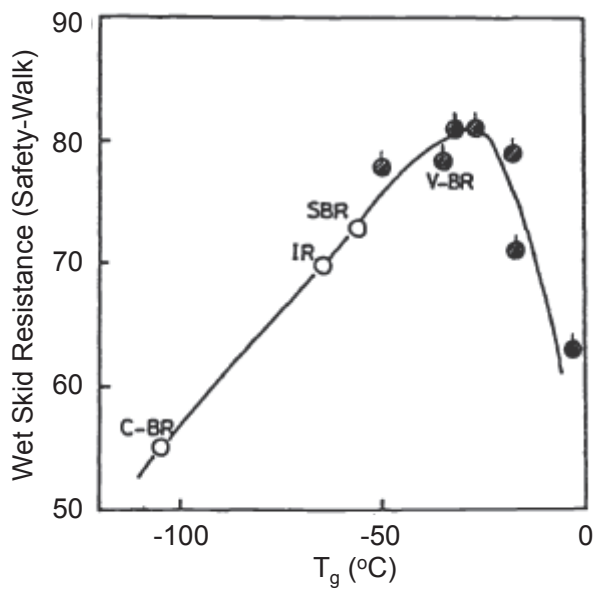

Fig. 16. Plot of wet skid resistance versus $T_{\mathrm{g}}$ : (०) several types of generalpurpose rubbers (cis-butadiene rubber (CBR), isoprene rubber (IR), SBR) and $(\bullet)$ butadiene rubber with various 1,2 unit contents. ${ }^{47)}$, Copyright (C) 1986, International Union of Pure and Applied Chemistry. because these conditions may reduce the strain energy per unit volume, which enhances the wear resistance. Additionally, wear resistance depends on the shape of the tire because the contact area between the tire and load is inversely proportional to the stress of tire; that is, the longer the width of the tire, the larger the area and the lower stress. Reducing stress may realize a high wear resistance.

\subsection{High Durability}

Durability is the ability to undergo a permanent deformation without cracking or fracturing and/or to withstand repeated deformation and recovery cycles. Because a low heat buildup, a low probability of cavitation, and suppression of crack growth are necessary, durability may rely on the dispersion of the filler into the rubber. ${ }^{51)}$ For instance, a homogeneous dispersion is preferred for high durability, but in practice, realizing a homogeneous dispersion is difficult. Consequently to form small flaws, a large deformation is applied prior to use because such flaws release the stress in the point of stress concentration. The internal structure, which supports the force loaded into filled rubber, is important to achieve a high durability.

\subsection{High Conductivity}

The conductivity and resistivity depend on the number of conductive paths from the top surface to the bottom surface in filled rubber. The volume resistivity of filled rubber decreases as the number of conducting paths increases. The abrupt decrease in the volume resistivity, i.e. a sudden increase in the number of the conductive paths, agrees well with the Percolation threshold of the filler in filled rubber. ${ }^{52)}$ The abrupt increase in the conductivity is associated with the formation of a filler network above the Percolation threshold. In particular, the volume resistivity of the filled natural rubber decreases suddenly at the Percolation threshold when carbon black is used as a filler. A non-uniform filler dispersion is necessary to form a filler network as conductivity paths.

\subsection{High Resistance to Thermal Aging}

Resistance to thermal aging is the most important subject for rubber used in harsh environments such as an automotive engine. It is important to note that the practical use of rubber becomes difficult when the resistance to thermal aging is inferior despite outstanding mechanical properties. Hence, fillers must have a superior resistance to thermal aging because most rubbers are easily degraded by heat.

Thermal aging begins when radicals are generated in the rubber. Radicals are used for rubbers with butadiene units to 
form carbon-carbon bonds and for rubbers with isoprene units to make chain scissions after reacting with oxygen. Therefore, rubbers with butadiene units become hard and brittle, whereas those with isoprene units become viscous and soft. Regardless, the filler must trap radicals to achieve a high resistance to thermal aging. Because carbon black consists of sp2 carbons with a long p-conjugation, it acts as a radical scavenger and traps radicals, efficiently improving the resistance to thermal aging. ${ }^{53)}$

\section{PERFORMANCE OF FILLED RUBBER}

The performance of filled rubber must be designed in accordance to the application with respect to the type of rubber, ingredients, recipe, and compounding method. Although many products, such as tires, engine mounts, belts, seismic isolation rubber, rubber rollers, etc. all use the same ingredients for compounding, the recipe and compounding method depend on the desired performance of the product.

\subsection{High-Performance Tires}

High-performance tires are designed to at least satisfy a high tensile strength, high tear strength, high rebound resilience, low heat buildup, and high wet skid resistance and so forth. ${ }^{54)} \mathrm{A}$ suitable size, the appropriate amount of filler uniformly dispersed into the rubber, and fillers bound to the rubber realize a high tensile strength, high tear strength, high rebound resilience, and low heat buildup, whereas controlling the glass transition temperature optimizes the wet skid resistance. The typical example is a silica-filled epoxidized natural rubber (e.g., ENASAVE $97^{55}$ ). The dispersivity and interfacial binding of nanosilica with natural rubber are greatly improved by introducing epoxy groups into natural rubber and using a suitable silane coupling agent. Furthermore, the epoxy group content adjusts the glass transition temperature of natural rubber. The high wet skid resistance of ENASAVE 97 is achieved at an epoxy group content of about $25 \mathrm{~mol} \%$.

\subsection{High Performance Engine Mounts}

High performance engine mounts are made of natural rubber due to the inherent properties of natural rubber ${ }^{56}$, such as a high tensile strength, high tear strength, high durability, low ratio of dynamic to static moduli, and high damping properties. Sometimes ethylene-propyrene-diene rubber, EPDM, is used to enhance the resistance to thermal aging because the resistance to thermal aging of EPDM is more prominent than that of natural rubber due to fewer carboncarbon double bonds in EPDM. High strengths and damping properties, which are antonymous, may be accomplished by precisely adjusting the size and amount of filler as well as its dispersivity in rubber. However, controlling the nanostructure may further improve the performance.

\subsection{High Performance Seismic Isolation Rubber}

The Great East Japan Earthquake in 2011 demonstrated that seismic isolation rubber is a rubber product that saves lives because it effectively protects buildings, houses, and architectural structures against earthquakes. To assure a high tensile strength, high tear strength, and high durability, seismic insulation rubber must be prepared from natural rubber with a suitable filler uniformly dispersed. ${ }^{57)}$ On the other hand, a specific non-uniform nanostructure is necessary for a high shear spring constant and high damping properties. Due to the discrepancies between the desired properties, both the abilities of the rubber and the filler must be exploited to serve distinct roles. Thus, the nanostructure should be well controlled in filled rubbers.

\subsection{High Performance Rubber Rollers}

Rubber rollers, which are useful for printing and copy machines, must exhibit a high tensile strength, high tear strength, and high conductivity. Although the strengths depend on the rubber and dispersion of the filler, using carbon black to form a filler network may increase the conductivity. ${ }^{58)}$ Thus, the nanostructure must be controlled precisely, and according to Percolation theory controlling the morphology is more important than controlling the filler network.

\section{SUMMARY}

To understand filled rubbers at the molecular level, their performances were divided into factors related to their properties. Because each product has distinct criteria, there is not a universal filled rubber for all applications. Consequently, how to enhance the performance of typical examples (i.e., tires, engine mounts, belts, seismic isolation rubbers, and rubber rollers) is discussed. Unfortunately a detailed exposition did not reveal a method to improve the overall performance of filled rubber. Because there are thousands of ways to control the performance of filled rubbers, research must focus on important factors specific to each product. In other words, filled rubber is an expression of the inventors and producer. When filled rubber is accepted as an everyday product, the engineers who developed the product will have achieved the ultimate success. 


\section{REFERENCES}

1) Donnet JB, Bansal RC, Wang MJ (eds), "Carbon Black: Science and Technology", (1993), Marcel Decker, New York, Hong Kong.

2) White JL, "Rubber Processing: Technology, Materials, Principles”, (1995), Carl Hanser Publishers, Munich.

3) Sato Y, Nippon Gomu Kyokaishi, 39, 185 (1966).

4) Sato Y, Nippon Goти Kyokaishi, 39, 420 (1966).

5) Guth E, Gold O, Phys Rev, 53, 322 (1938).

6) Smallwood HM, J Appl Phys, 15, 758 (1944).

7) Guth E, J Appl Phys, 16, 20 (1945).

8) Halpin JC, Compos Mater, 3, 732 (1969).

9) Merckel Y, Diani J, Brieu M, Caillard J, J Appl Polym Sci, 129, 2086 (2013).

10) Fukahori Y, Nippon Gomu Kyokaishi, 77, 18 (2004).

11) Rivlin D, Aron J, Medalia AL, Rubber Chem Technol, 41, 330 (1968).

12) Watson JW, Trans I R I, 32, 204 (1956).

13) Kraus G, Gruver JT, Rubber Chem Technol, 41, 1256 (1968).

14) Nishi T, J Polym Sci, Polym Phys Ed, 12, 685 (1974).

15) Choi SS, J Appl Polym Sci, 93, 1001 (2004).

16) Sirisinha C, Sittichokchuchai W, J Appl Polym Sci, 76, 1542 (2000).

17) Robertson CG, Lin CJ, Rackaitis M, Roland CM, Macromolecules, 41, 2727 (2008).

18) Klüppel M, Adv Polym Sci, 164, 1 (2003).

19) Klüppel M, Heinrich G, Kautsch Gummi Kunst, 58, 217 (2005).

20) Robertson CG, Lin CJ, Rackaitis M, Roland CM, Macromolecules, 41, 2727 (2008).

21) Isono $\mathrm{Y}$, Satoh $\mathrm{Y}$, Fujii $\mathrm{S}$, Kawahara $\mathrm{S}$, Kagami S, Adv Mater Res, 11-12, 729 (2006).

22) Satoh $Y$, Suda K, Fujii S, Kawahara S, Isono Y, Kagami Y, e- $J$ Soft Mater, 3, 14 (2007).

23) Payne AR, J Appl Polym Sci, 7, 873 (1963).

24) Mullins L, Rubber Chem Technol, 42, 339 (1969).

25) Gent AN, Park BJ, J Mater Sci, 19, 1947 (1984).

26) Cho K, Gent AN, J Mater Sci, 23, 141 (1988).

27) Cho K, Gent AN, Lam PS, J Mater Sci, 22, 2899 (1987).

28) Oberth AE, Bruenner RS, Trans Soc Rheol, 9, 165 (1965).

29) Oberth AE, Rubber Chem Technol, 40, 1337 (1967).

30) Gent AN, Lindley PB, Proc R Soc London A, Math Phys Sci, 249, 195 (1959).

31) Hess WM, Lyon F, Burgess K, Kautsch Gummi Kunst, 20, 135 (1967).

32) Zhang H, Scholz AK, de Crevoisier J, Vion-Loisel F, Besnard G, Hexemer A, Brown HR, Kramer EJ, Creton C, Macromolecules, 45, 1529 (2012).
33) Kato A, Ikeda Y, Tsushi R, Kokubo Y, Kojima N, Colloid Polym Sci, 291, 2101 (2013).

34) Kawahara S, Yamamoto Y, Fujii S, Isono Y, Niihara K, Jinnai H, Nishioka H, Takaoka A, Macromolecules, 41, 4510 (2008).

35) Kosugi K, Sutthangkul R, Chaikumpollert O, Yamamoto Y, Sakdapipanich J, Isono Y, Kawahara S, Colloid Polym Sci, 290, 1457 (2012).

36) Kawahara S, Chaikumpollert O, Akabori K, Yamamoto Y, Polym Adv Technol, 22, 2665 (2011).

37) Chaikumpollert O, Yamamoto Y, Suchiva K, Kawahara S, Colloid Polym Sci, 290, 331 (2012).

38) Greensmith HHW, J Polym Sci, 18, 189 (1955).

39) Noguchi F, Akabori K, Yamamoto Y, Kawazura T, Kawahara S, Kobunshi Ronbunshu, 66, 454 (2009).

40) Thomas AG, Wittle JM, Rubber Chem Technol, 43, 222 (1970).

41) Sambrook RW, Rubber Chem Technol, 44, 728 (1971).

42) Glucklich J, Landel RF, J Appl Polym Sci, 20, 121 (1976).

43) Rivlin RS, Thomas AG, J Polym Sci, 10, 291 (1953).

44) Chun H, Gent AN, Rubber Chem Technol, 69, 577 (1996).

45) Akutagawa K, Yamaguchi K, Yamamoto A, Heguri H, Jinnai H, Shinbori Y, Rubber Chem Technol, 81, 182 (2008).

46) Ward IM, Hadley DW, "An Introduction to the Mechanical Properties of Solid Polymers", (1996), John Wiley \& Sons, Chichester.

47) Yoshioka A, Komuro K, Ueda A, Watanabe H, Akita S, Masuda T, Nakajima A, IUPAC Pure Appl Chem, 58, 1697 (1986).

48) Gal AL, Yang X, Klüppel M, J Chem Phys, 123, 014704 (2005).

49) Russell W, Rubber World, 217, 33 (1997).

50) Gent AN, Rubber Chem Technol, 62, 750 (1989).

51) Soltani S, Sourki FA, Ir Polym J, 14, 745 (2005).

52) McQueen DH, Jäger KM, Pelíšková M, J Phys D: Appl Phys, 37, 2160 (2004).

53) Levy M, Kautsch Gummi Kunst, 42, 129 (1989).

54) Haney P, "The Racing \& High-Performance Tire: Using Tires to Tune for Grip \& Balance", (2003), Society of Automotive Engineers, Inc., Warrendale.

55) Wada T, Nippon Gomu Kyoukaishi, 85, 183 (2012).

56) Ngolemasango FE, Bennett M, Clarke J, J Appl Polym Sci, 110, 348 (2008).

57) Bhuiyan AR, Okui Y, Mitamura H, Imai T, Int J Sol Struct, 46, 1778 (2009).

58) Kim K, Kim CH, Kim HY, Kim DS, Jpn J Appl Phys, 49 05EC04 (2010). 